\title{
Pronóstico de Oportunidades de Espectro Radioeléctrico en una Red Móvil con base en el Modelo de Propagación Interino de la Universidad de Stanford
}

\author{
Luis F. Pedraza*, Cesar A. Hernández y Juan M. Sanchez \\ Universidad Distrital Francisco José de Caldas, Facultad de Tecnologia e Ingenieria, Carrera 7 No. $40 \mathrm{~B}-$ \\ 53, Bogotá-Colombia (e-mail: Ifpedrazam@udistrital.edu.co; cahernandezs@udistrital.edu.co; \\ jmsanchezc@udistrital.edu.co)
}

${ }^{*}$ Autor a quien debe ser dirigida la correspondencia

Recibido Oct. 24, 2017; Aceptado Dic. 27, 2017; Versión final Ene. 23, 2018, Publicado Ago. 2018

\begin{abstract}
Resumen
Se presenta el desarrollo de un método que identifica las oportunidades de espectro radioeléctrico en un canal de una red celular móvil para un entorno urbano usando el pronóstico de potencia de la señal recibida. El pronóstico de ocupación de espectro radioeléctrico ha demostrado ser útil en el diseño de sistemas inalámbricos capaces de aprovechar las oportunidades de espectro, como la radio cognitiva, que es una de las tecnologías que se incluye en el Internet de las cosas. El método propuesto integra el modelo de propagación a gran escala Interino de la Universidad de Stanford con un modelo neuronal de onda wavelet. Los resultados del método son consistentes con el comportamiento observado en experimentos realizados en sistemas inalámbricos de este tipo. El modelado desarrollado en el canal de la banda del sistema global para las comunicaciones móviles puede contribuir a que los usuarios de radio cognitiva compartan canales y eviten colisiones.
\end{abstract}

Palabras clave: espectro radioeléctrico; modelo neuronal wavelet; modelo Interino; radio cognitiva; ciclo de trabajo

\section{Radioelectric Spectrum Opportunities Forecast in a Mobile Network based on the Stanford University Interim Propagation Model}

\begin{abstract}
The development of a method that identifies radioelectric spectrum opportunities in a channel of a mobile cellular network for an urban environment using received signal power forecast is presented. Radioelectric spectrum occupancy forecast has proven useful in the design of wireless systems able to harness spectrum opportunities like cognitive radio, which is one of the technologies included in the Internet of things. The proposed method integrates the Stanford University Interim large-scale propagation model with a wavelet neural model. The results obtained through simulations are consistent with the observed behavior in experiments carried out in wireless systems of this type. The proposed model in the channel of global system for mobile communication band may help cognitive radio users to share channels and to avoid collisions.
\end{abstract}

Keywords: radioelectric spectrum; wavelet neural model; interim model; cognitive radio; duty cycle 


\section{INTRODUCCIÓN}

El espectro radioeléctrico se percibe como un bien escaso. En la actualidad, el uso de un gran porcentaje de las bandas licenciadas es bajo, normalmente se pueden encontrar bandas de frecuencia muy congestionadas y otras bandas poco utilizadas (López et al., 2015). La Radio Cognitiva (CR, por sus siglas en inglés: Cognitive radio) se ha convertido en uno de los paradigmas más investigados en las comunicaciones de radio para optimizar el uso del espectro radioeléctrico (Haykin, 2005; Pedraza et al., 2016a). Una CR es una radio inteligente consciente del contexto, que es capaz de reconfigurarse de manera autónoma para aprender y adaptarse al entorno de radio que la rodea (Akyildiz et al., 2006; Hernández et al., 2015). La investigación en CR ha sido motivada por los resultados de las campañas de medición de espectro realizadas en todo el mundo (Sanders, 1997; Chiang et al., 2007; Chen et al., 2009; Lopez y Casadevall, 2010; Wellens y Mahonen, 2010; Patil et al., 2011; Rocke y Wyglinski, 2011; Taher et al., 2011; Pedraza et al., 2013; Al-Hourani et al., 2015; Mehdawi et al., 2015). Estas campañas de medición muestran que el espectro radioeléctrico está subutilizado en los dominios de frecuencia, tiempo y espacio geográfico (Chiang, et al., 2007; Lopez y Casadevall, 2010; Wellens y Mahonen, 2010; Patil et al., 2011; Taher et al., 2011; Pedraza et al., 2013). El principio para el funcionamiento de la CR se basa en que los usuarios no licenciados no interfieran a los usuarios licenciados. Una forma de enfrentar este problema es que los usuarios no licenciados detecten la ocupación del espectro radioeléctrico en diferentes localizaciones como función del entorno considerado y de las condiciones de propagación, lo cual proporciona una valiosa herramienta para el diseño, dimensionamiento y evaluación del rendimiento en redes de CR (Lopez y Casadevall, 2011; Galeano et al., 2016).

Los modelos de propagación comenzaron a formularse a finales de la década de los años sesenta, con el fin de estimar con precisión las pérdidas de propagación en un entorno. Inicialmente, se diseñaron modelos de propagación empíricos y estadísticos en áreas urbanas (Okumura et al., 1968; Turin et al., 1972). Luego, con el despliegue de las comunicaciones móviles, a comienzos de la década de los ochenta, se diseñaron modelos de propagación para escenarios de micro y macroceldas (Hata, 1980; Walfisch y Bertoni, 1988; Har, et al., 1999). A partir de esto, han surgido numerosos esfuerzos para entender y pronosticar las características de los canales en las comunicaciones móviles (Sarkar et al., 2003). Las series de tiempo han sido empleadas, en algunos casos, como mecanismo para el pronóstico de las pérdidas de propagación. Por ejemplo, se han utilizado las redes neuronales para pronosticar la intensidad de campo (Stocker et al., 1993), así como las perdidas promedio de propagación (Ostlin et al., 2010; Sotiroudis et al., 2013), y con base en la integración con los modelos de Okumura-Hata (Pedraza et al., 2016e; Pedraza et al., 2017) y COST 231 Walfisch-Ikegami (Pedraza et al., 2017a). Otro método utilizado es la lógica difusa (Phaiboon et al., 2005). En este artículo se desarrolla un pronóstico de la potencia recibida con el fin de identificar las oportunidades de espectro radioeléctrico dentro de una red móvil licenciada que integra el modelo de propagación Interino de la Universidad de Stanford (SUI, por sus siglas en inglés: Stanford University Interim) con el modelo neuronal wavelet en un entorno urbano.

\section{MÉTODO PROPUESTO}

Inicialmente, se realiza el diseño del modelo neuronal wavelet, que permite pronosticar la potencia instantánea. Posteriormente, se ajusta el modelo de propagación SUI a partir de las medidas obtenidas alrededor del entorno urbano, con este modelo se obtiene la potencia de recepción promedio. Finalmente, se presenta la metodología propuesta que integra los modelos indicados anteriormente y pronostica las oportunidades de espectro radioeléctrico.

\section{Modelo neuronal wavelet}

Para sistemas cognitivos en (Bai et al., 2014) se hace uso de una red neuronal de propagación hacia atrás para predecir el estado del espectro, y en (Kunwei et al., 2014; Bai et al., 2015) se optimiza la red neuronal con un algoritmo genético. También, en (Iliya et al., 2014) una red neuronal pronostica la potencia en las bandas de televisión y GSM900. Los ejemplos anteriores y lo descrito en (Pedraza et al., 2016b) demuestran el carácter promisorio de las redes neuronales en el pronóstico de la potencia recibida en canales inalámbricos, sobre modelos como Markov y de descomposición de modo empírico-vector de soporte para regresión. Por tanto, en este artículo se propone el uso de una teoría que combina las disciplinas wavelet y redes neuronales (Zhang y Benveniste, 1992), para pronosticar la potencia recibida en un canal de la tecnología sistema global para las comunicaciones móviles (GSM).

La señal de entrada al modelo, que corresponde a la potencia recibida en un canal GSM para las mediciones realizadas en (Pedraza et al., 2014) y analizadas en (Pedraza et al., 2016b), se descompone usando la wavelet madre Discrete Meyer (dmey), que presentó un menor error al ser comparada con las wavelets madre Daubechies, Coiflets y Symlets (Debnath y Shah, 2014). El resultado es dos niveles que 
contienen en total cuatro coeficientes. Estos coeficientes se envían a la entrada de la red neuronal wavelet multi-capa de propagación hacia atrás mostrada en la Fig. 1, y expresada como (Pedraza, 2016):

$$
f[n]=g \sum_{i=1}^{n}\left[\frac{1}{\sqrt{M}} \sum_{k} W_{\Phi}\left[j_{0}, k\right] \Phi_{j o, k}[n]+\frac{1}{\sqrt{M}} \sum_{j=j_{0}}^{\infty} \sum_{k} W_{\psi}[j, k] \psi_{j, k}[n]\right]
$$

donde $g$ es la función de activación de la red neuronal, que para este caso contiene 2 entradas, 2 salidas y 2 capas ocultas. La red fue entrenada en un inicio con aproximadamente cinco días de mediciones continuas y se aumentó el número de patrones de entrenamiento hasta que el error disminuyó y se hizo relativamente constante, esto se alcanzó para 1000 patrones de entrenamiento. Finalmente, la salida de la red neuronal es reconstruida usando un análisis wavelet para obtener la potencia pronosticada, cuyo tiempo de entrenamiento basta ser de un día para alcanzar un error cuadrático medio de 2.9262 y con un incremento menor al $10 \%$ respecto al entrenamiento de cinco días; tal como se indica en (Pedraza et al., 2016b; Pedraza et al., 2016c; Pedraza et al., 2016d).

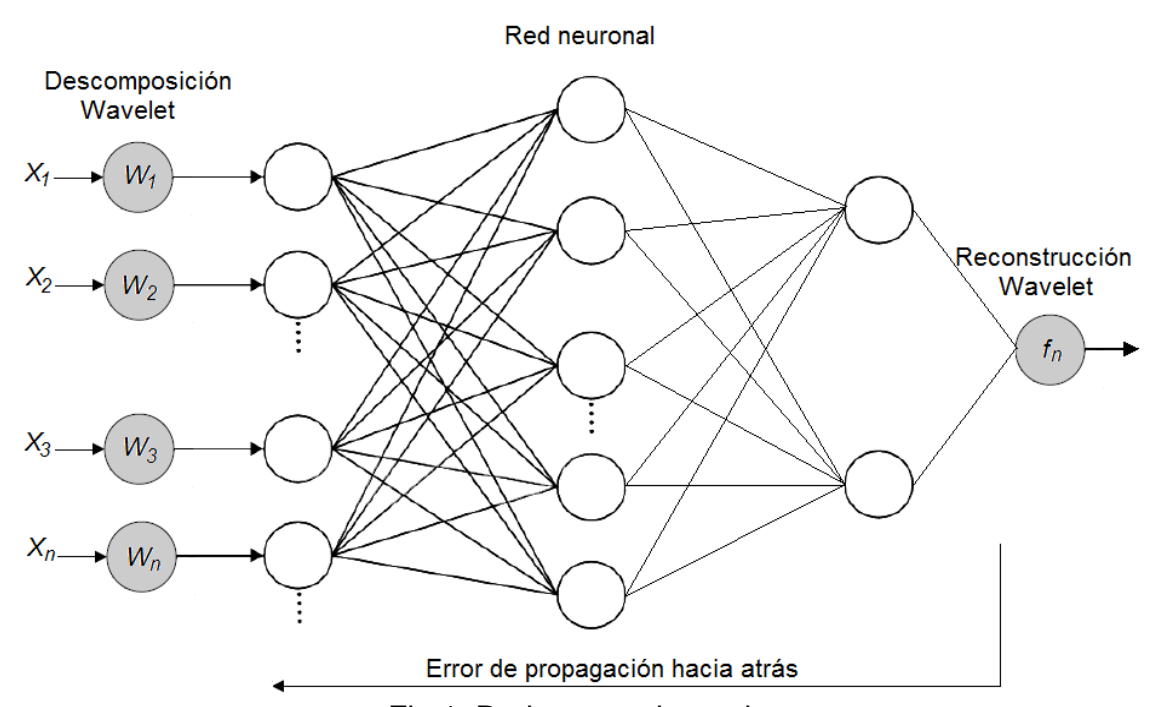

Fig.1: Red neuronal wavelet

\section{Modelo Interino de la Universidad de Stanford}

En la Fig. 2 se presenta el entorno alrededor de la celda de la estación base (BS, por sus siglas en inglés: Base station) en el que se realizaron mediciones con el analizador de espectro para el norte de la ciudad de Bogotá-Colombia. Los seis puntos de medida corresponden a sitios de cobertura de la celda, que se encuentran a diferentes distancias de la BS, para evaluar y ajustar el modelo de propagación SUI (Molisch, 2011). El tiempo de duración de las medidas en estos seis puntos fue aproximadamente de una hora. El entorno es llano y está conformado en su mayoría por concentración de edificios; aunque también hay zonas verdes, con algunos árboles, como se observa en el punto de medida D. En la Tabla 1 se presentan los parámetros del transmisor y receptor para evaluar el modelo de propagación SUI. A continuación se ajusta el modelo tomando como referencia las potencias medidas en los seis puntos de la Fig. 2.

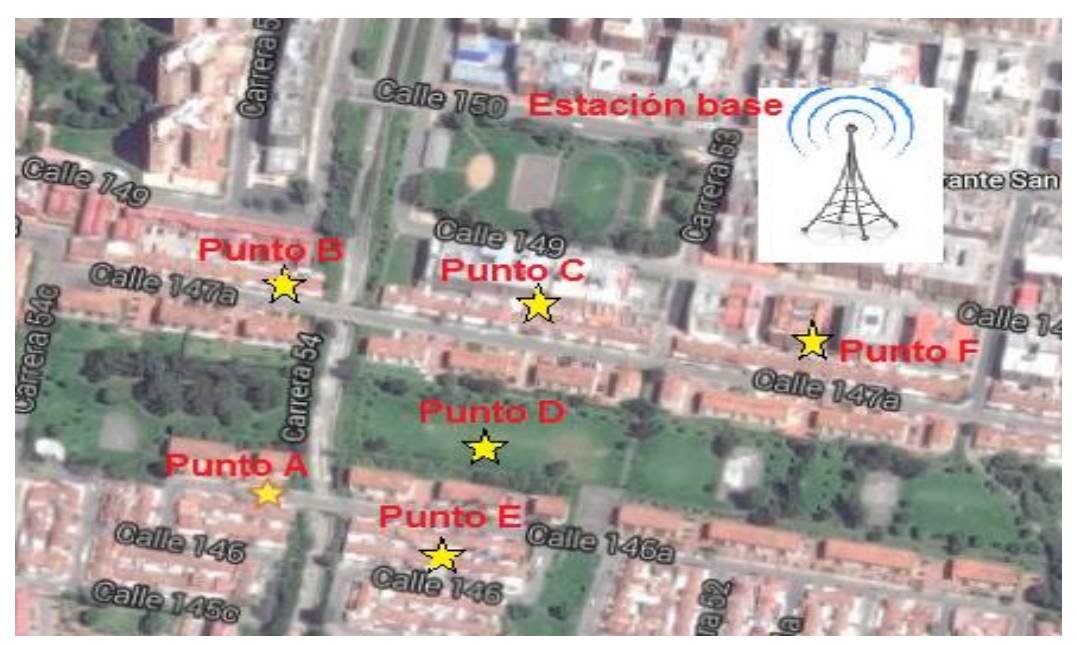

Fig. 2: Puntos de medida en la zona norte de la ciudad de Bogotá-Colombia 
Tabla 1: Parámetros de la BS y el analizador de espectro

\begin{tabular}{|c|c|}
\hline Parámetro & Valor \\
\hline Potencia de transmisión $\mathrm{BS}\left(\mathrm{P}_{\mathrm{Tx}}\right)$ & $26 \mathrm{dBm}$ \\
\hline Altura BS & $26 \mathrm{~m}$ \\
\hline Ganancia antena BS (Gвтs) & $16.5 \mathrm{dBi}$ \\
\hline Perdidas combinador BS ( $\left.\mathrm{L}_{c o}\right)$ & $4 \mathrm{~dB}$ \\
\hline Perdidas cable BS $\left(L_{c}\right)$ & $4 \mathrm{~dB}$ \\
\hline Ganancia antena analizador ( $\left.\mathrm{G}_{\mathrm{An}}\right)$ & $3 \mathrm{dBi}$ \\
\hline Perdidas cable analizador $\left(\mathrm{L}_{c a}\right)$ & $0.72 \mathrm{~dB}$ \\
\hline Ganancia Amplificador de bajo ruido (GLNA) & $11 \mathrm{~dB}$ \\
\hline Altura analizador & $1.5 \mathrm{~m}$ \\
\hline Frecuencia de transmisión canal GSM & $828.93 \mathrm{MHz}$ \\
\hline
\end{tabular}

Haciendo uso de la ecuación (2) se obtienen las pérdidas de propagación teóricas promedio $(\bar{L})$ del modelo SUI para cada punto de medida, como se observa en la Tabla 2,

$\bar{L}(d B)=A+10 \gamma \log \left[\frac{d}{d_{0}}\right]+S$

donde,

$$
\begin{gathered}
A=20 \log \left[\frac{4 \pi d_{0}}{\lambda}\right] \\
\gamma=a-b h_{b}+\frac{c}{h_{b}}
\end{gathered}
$$

$d$ es la distancia entre el transmisor y el receptor en metros, $d_{0}=100 \mathrm{~m}, h_{b}$ es la altura de la BS en metros, $\lambda$ es la longitud de onda en metros, $S$ es el efecto de sombreado y $\gamma$ es el exponente de pérdidas por trayectoria. Las constantes $a, b$ y $c$ dependen de la categoría del terreno y se calculan para un terreno tipo $B$ según (Erceg et al., 1999).

Tabla 2: Pérdidas de propagación del modelo

SUI para los puntos de medida de la Fig. 2

\begin{tabular}{|c|c|}
\hline Punto & $\bar{L}$ canal GSM (dB) \\
\hline $\mathrm{F}(58 \mathrm{~m})$ & 69.793 \\
\hline $\mathrm{C}(152 \mathrm{~m})$ & 88.574 \\
\hline $\mathrm{D}(226 \mathrm{~m})$ & 96.306 \\
\hline $\mathrm{B}(287 \mathrm{~m})$ & 100.965 \\
\hline $\mathrm{E}(290 \mathrm{~m})$ & 101.167 \\
\hline $\mathrm{A}(328 \mathrm{~m})$ & 103.568 \\
\hline
\end{tabular}

Con los resultados de la Tabla 2 y los parámetros de la Tabla 1, se obtiene la potencia de recepción promedio $\left(\overline{P_{R X}}\right)$ teórica (Rappaport, 2002):

$\overline{P_{R X}}=P_{T X}+G_{B T S}+G_{A n}+G_{L N A}-\bar{L}-L_{c}-L_{c o}-L_{c a}$

En la Fig. 3 se presenta la $\overline{P_{R X}}$ hallada a través de la ecuación (5) para el modelo SUI en comparación al rango de la potencia de recepción medida con sus respectivos valores promedio. En la Fig. 3 existe una diferencia apreciable entre los datos teóricos respecto a los medidos. 


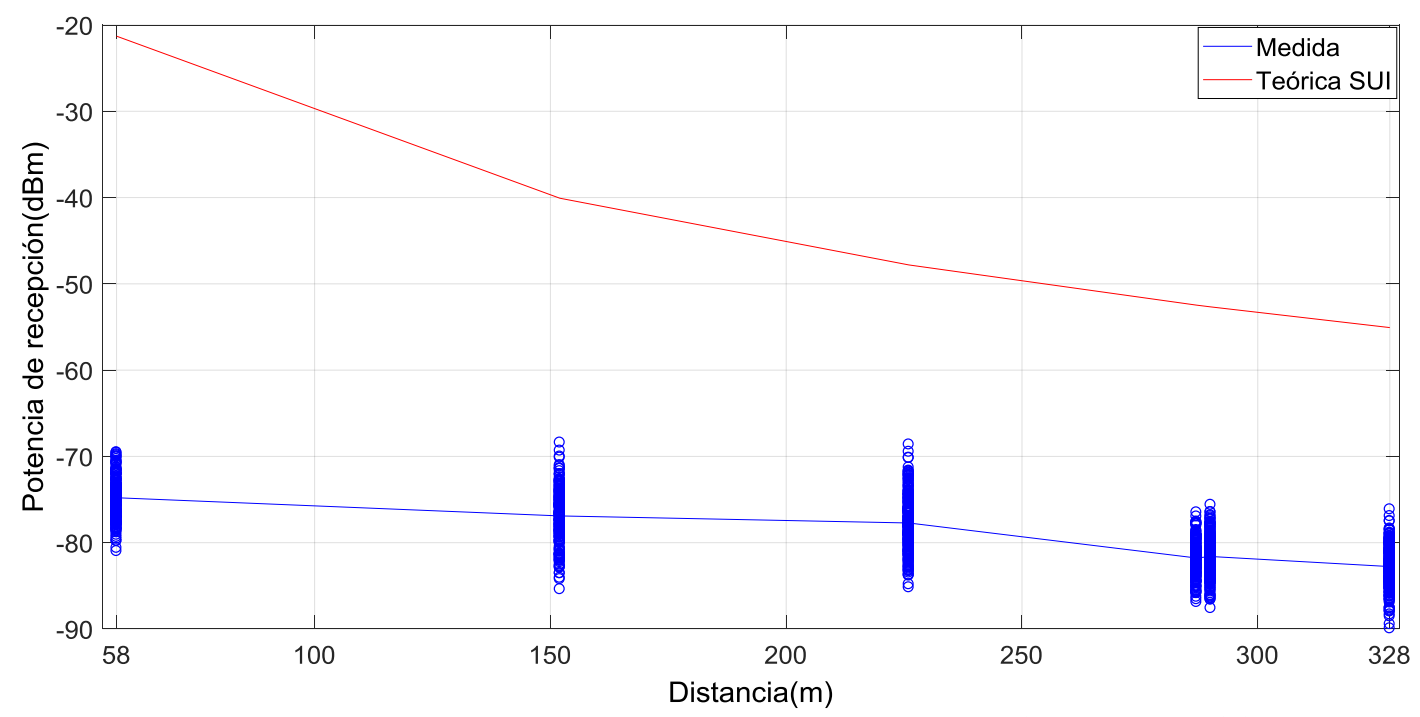

Fig. 3: Potencia de recepción teórica y medida para el modelo SUI del canal GSM

Con el fin de aumentar la precisión del modelo de propagación SUI, se usa el método de mínimos cuadrados (Madsen et al., 2004), para ajustar los resultados teóricos a los valores promedio medidos. A partir de esto se obtiene la siguiente ecuación ajustada para el canal GSM:

$\bar{L}(d B)=A+2.287 \gamma \log \left[\frac{d}{d_{0}}\right]+S+44.3754$

En la Fig. 4 se presenta la potencia de recepción del modelo SUI ajustado con la ecuación (6), respecto al promedio de la potencia de recepción medida. En la Fig. 4 se aprecia una aproximación entre los valores medidos y el modelo ajustado, cuyo error cuadrático medio es 1.2688.

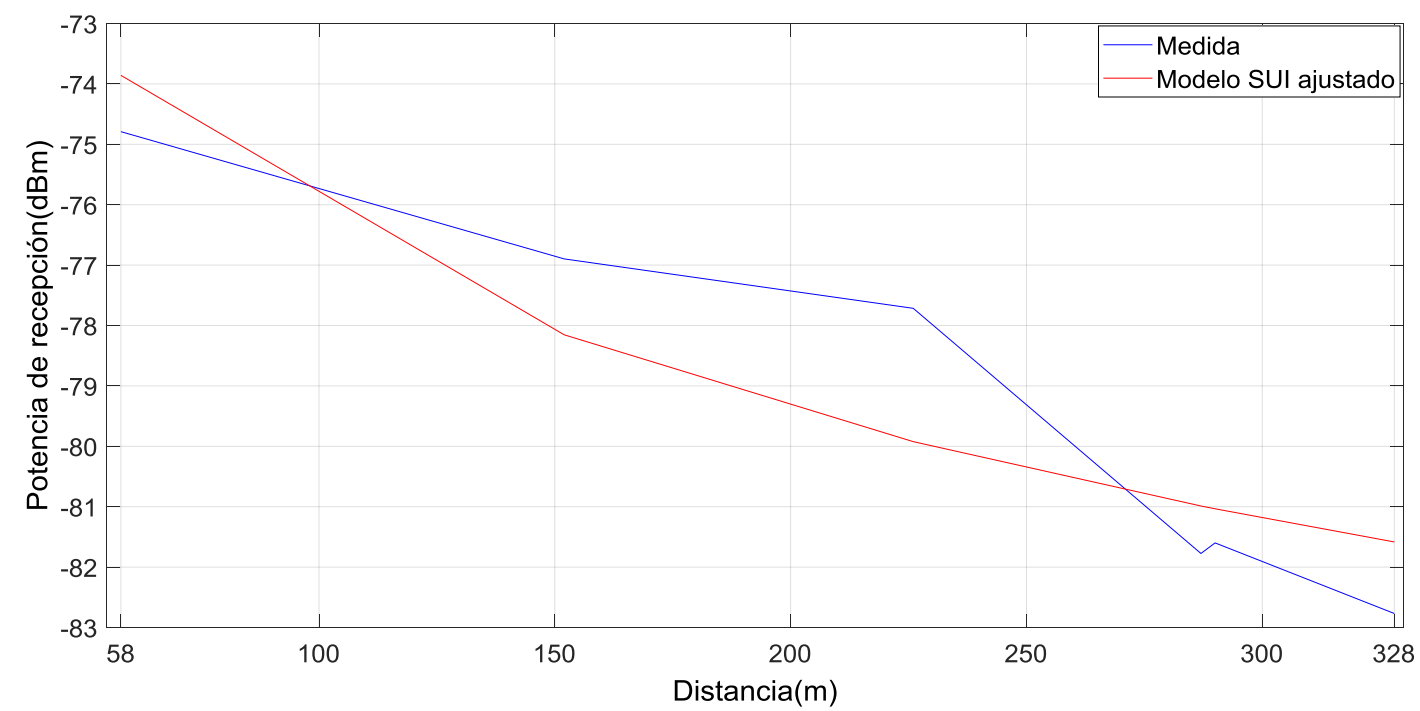

Fig. 4: Potencia de recepción ajustada y medida para el modelo SUI del canal GSM

\section{Metodología propuesta}

A continuación se describe la metodología usada para desarrollar el método propuesto, y la ecuación general para pronosticar la potencia de recepción con el modelo neuronal wavelet y el modelo de propagación SUI ajustado anteriormente. El procedimiento general para obtener el método que pronostica las oportunidades de espectro radioeléctrico en un entorno desconocido es el siguiente (Pedraza, 2016):

Paso 1. Realizar mediciones: En este paso se toman las mediciones del canal y/o canales del espectro radioeléctrico variantes en función del tiempo durante un día, como se describe en (Pedraza et al., 2014); Paso 2. Ajustar el modelo de propagación: El modelo de propagación SUI se ajusta, por ejemplo con el método de mínimos cuadrados, en función de los valores promedio de las mediciones; Paso 3. Entrenar 
modelo neuronal wavelet: Con las mediciones realizadas por un tiempo mínimo de 24 horas se entrena el modelo neuronal wavelet diseñado; Paso 4. Integrar modelos: Extrapolar el modelo de propagación SUI ajustado al modelo neuronal wavelet, integrando las pérdidas de propagación promedio con las perdidas instantáneas; y Paso 5. Pronosticar la potencia de recepción: A lo largo del entorno urbano analizado se pronostica la potencia de recepción durante un tiempo determinado, con base en el modelo compuesto en el paso 4.

Por consiguiente, el método que tiene en cuenta las pérdidas instantáneas como las pérdidas promedio de propagación, se puede escribir a nivel general de la siguiente forma:

$L=\Delta L+\bar{L}$

donde $\Delta L=\left.f(f[n])\right|_{f[n]=\Delta P R X}$ son las pérdidas de propagación instantáneas en función de la potencia de recepción obtenida en la ecuación (1), y $\bar{L}$ son las pérdidas de propagación promedio obtenidas a partir del ajuste de la ecuación (2) del modelo SUI. Por tanto, al combinar las ecuaciones (1) y (5), se obtiene la potencia de recepción en función del modelo SUI:

$P_{R X}=g \sum_{i=1}^{n}\left[\frac{1}{\sqrt{M}} \sum_{k} W_{\Phi}\left[j_{0}, k\right] \Phi_{j o, k}[n]+\frac{1}{\sqrt{M}} \sum_{j=j_{0}}^{\infty} \sum_{k} W_{\psi}[j, k] \psi_{j, k}[n]\right]+\overline{P_{R X}}$

donde $\overline{P_{R X}}=f(\bar{L})$. En la Fig. 5 se representa la ecuación (8).

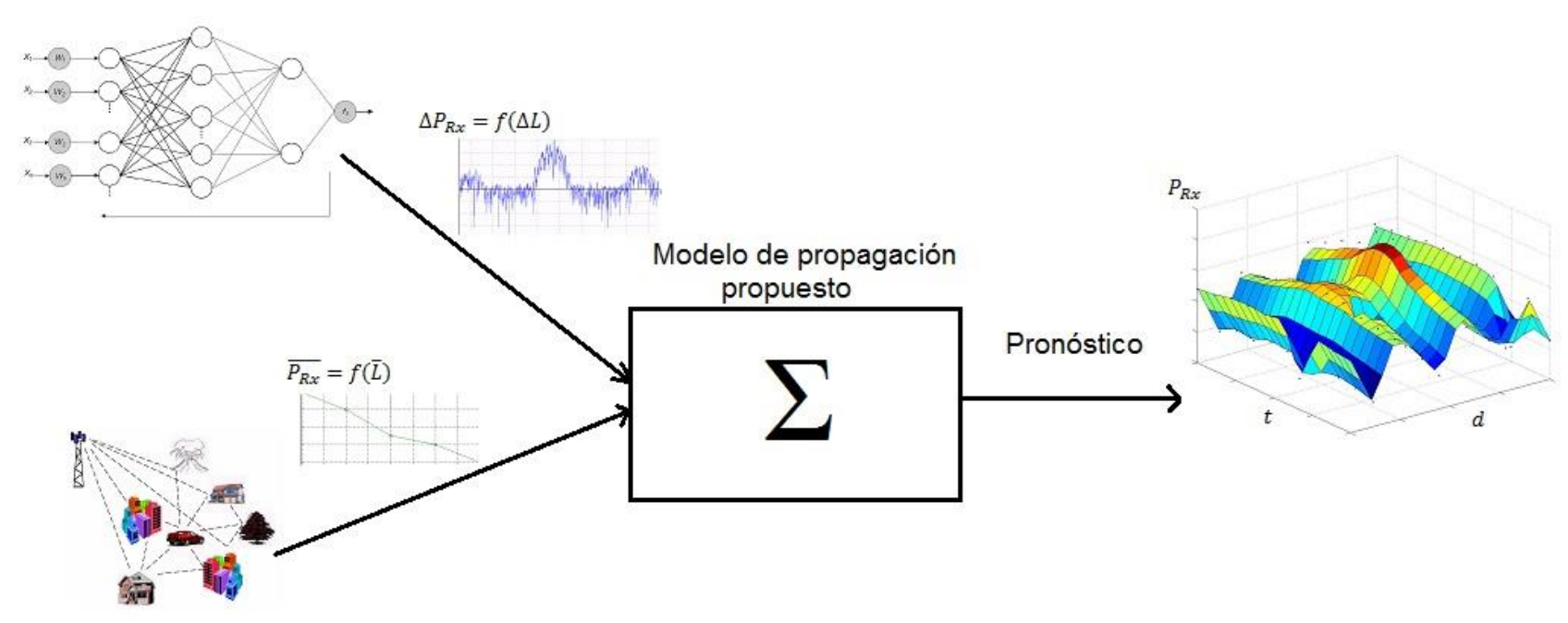

Fig. 5: Esquema del método propuesto para pronosticar las oportunidades espectrales

\section{RESULTADOS Y DISCUSIÓN}

En esta sección se presentan y discuten los resultados del método propuesto en la sección anterior para los diferentes niveles de ocupación de los canales. En la Fig. 6 se exhibe un ejemplo en el que existe aplicación del método propuesto, aquí el usuario de CR percibe la potencia de una BS primaria y se puede desplazar a través de la cobertura de la celda como lo indica la dirección de las flechas. El usuario de CR puede pronosticar el nivel de potencia que recibirá de la BS primaria a diferentes distancias y teniendo en cuenta las pérdidas de propagación del entorno. Los resultados fueron analizados usando el software Matlab® en un computador de dos núcleos de procesamiento de $2.4 \mathrm{GHz}$ y $4 \mathrm{~GB}$ de RAM. La evaluación del método propuesto realiza el pronóstico de hasta en una hora de la potencia de recepción; con una distancia máxima de $328 \mathrm{~m}$. También, se presenta el ciclo de trabajo sobre el entorno analizado.

A partir de la ecuación (8), la ecuación del método propuesto en el entorno de la Fig. 2 es la ecn. (9) que es graficada en la figura 7 :

$P_{R X}=g \sum_{j_{0}=1}^{n}\left[\frac{1}{\sqrt{M}} \sum_{k} W_{\Phi}\left[j_{0}, k\right] \Phi_{j o, k}[n]+\frac{1}{\sqrt{M}} \sum_{j=j_{0}}^{\infty} \sum_{k} W_{\psi}[j, k] \psi_{j, k}[n]\right]+f\left[A+2.287 \gamma \log \left[\frac{d}{d_{0}}\right]+S+44.3754\right]$ 


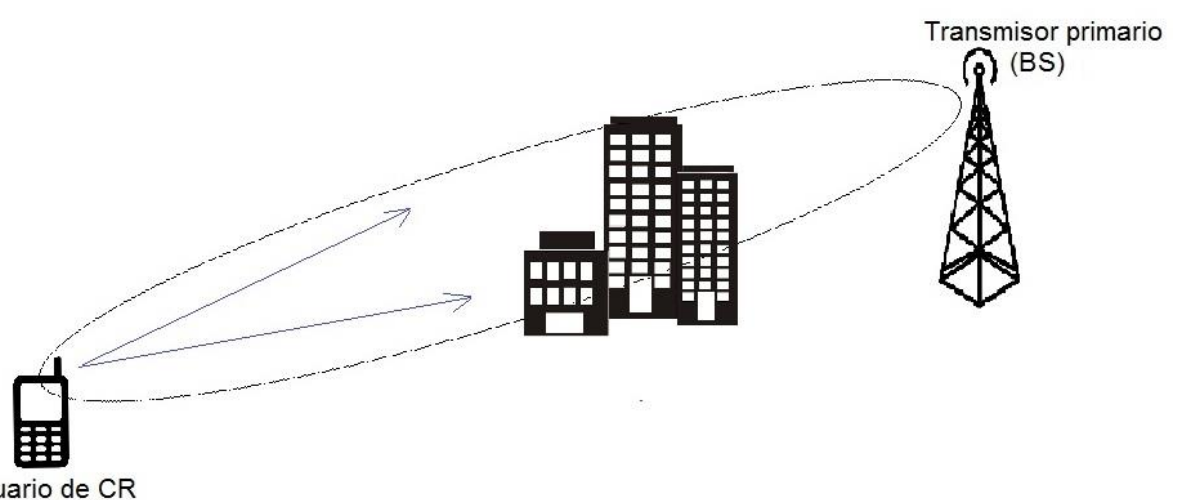

Fig. 6: Ejemplo de aplicación para el método propuesto

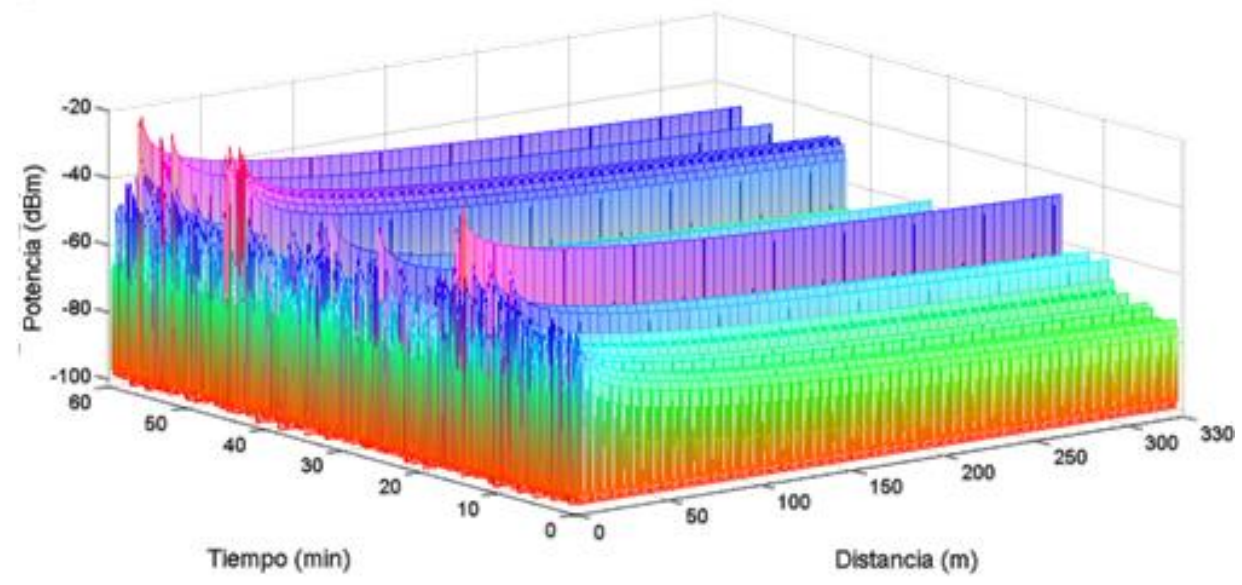

Fig. 7: Potencia de recepción para el método propuesto

En la Fig. 7 se identifican las oportunidades de espectro en color naranja, que serían percibidas y aprovechadas por parte de los usuarios de CR; aunque para ser más preciso se depende del umbral seleccionado, que para este caso es $-89 \mathrm{dBm}$. Estas son obtenidas del pronóstico de una hora de potencia con base en la información histórica de un día. En la Fig. 7 el nivel de potencia tiene la tendencia a disminuir a medida que aumenta la distancia, según las pérdidas halladas. En el ejemplo de la Fig. 6, el análisis del método propuesto es realizado para desarrollar el pronóstico de la potencia desde el usuario de CR, haciendo un símil con el analizador de espectro en el que se efectuaron las mediciones; sin embargo esto depende de la arquitectura de CR desplegada en el entorno. Debido a que el procesador y el consumo de energía son más limitados en el equipo del usuario de CR, se recomienda el uso de una arquitectura con infraestructura donde el pronóstico se realice desde la BS de CR. La BS de CR es provista con un mejor procesador respecto al del usuario de $\mathrm{CR}$ y sin limitaciones en el consumo de energía. No obstante, el tiempo entre la captura de datos en el entorno y el procesamiento agrega un retraso en la respuesta que no debe ser ignorado; pero el pronóstico ayuda a reducir el impacto negativo del retraso en la respuesta. En la Fig. 8 se presenta una arquitectura con infraestructura (Akyildiz et al., 2006).

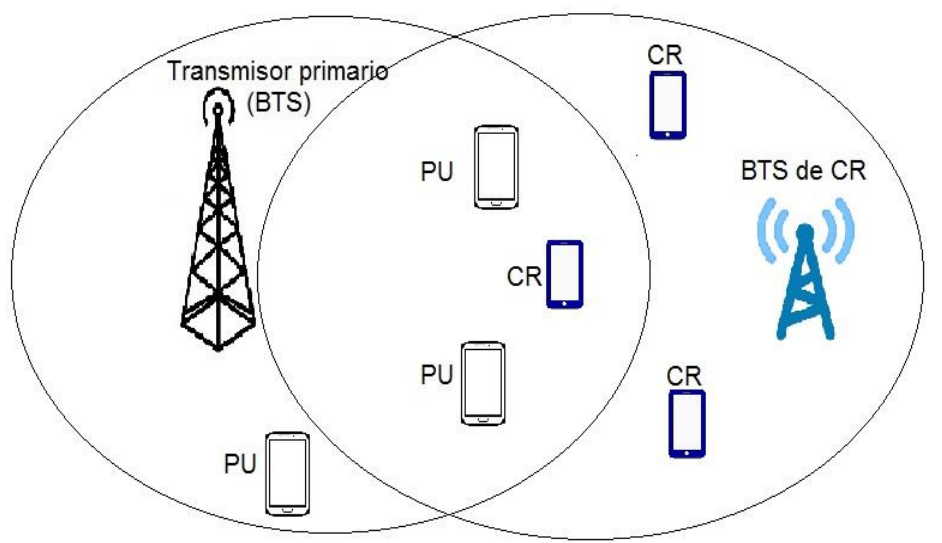

Fig. 8: Arquitectura de CR con infraestructura 
Para un sistema de CR, el modelamiento desarrollado en el canal de la banda GSM, puede contribuir a mejorar el uso de la eficiencia espectral, puesto que permitiría a los usuarios de CR compartir los canales y evitar las colisiones con los usuarios primarios en las oportunidades de espectro radioeléctrico encontradas.

\section{Ciclo de trabajo}

El pronóstico del ciclo de trabajo se encuentra a partir de la ecuación (10) (Lopez y Casadevall, 2011; Lopez y Casadevall, 2017):

$\psi=\left(1-\sum_{k=1}^{K} \alpha_{k}\right) P_{f a}+\sum_{k=1}^{K} \alpha_{k} Q\left(\frac{Q^{-1}\left(P_{f a}\right) \sigma_{N}-\gamma_{k}}{\sigma_{S k}}\right)$

donde $\mathrm{K}>0$ representa el número de niveles de potencia de transmisión que pueden estar presentes en el canal, para este caso en las medidas del punto A de la Fig. 2 existe una sola potencia de transmisión. 0 $<\alpha_{k} \leq 1$ es el factor de actividad del k-ésimo nivel de potencia, esto se obtiene del valor promedio de uso del canal GSM analizado. Pfa es la probabilidad de falsa alarma objetivo considerada para la selección del umbral de decisión de la energía, en este ejercicio es de $1 \% \cdot \gamma_{k}=P_{R X k}-P_{N}$ es la relación señal a ruido que resulta del k-ésimo nivel de potencia de transmisión promedio. $\sigma_{S k}$ y $\sigma_{N}$ son la desviación estándar en decibelios de los k-ésimos niveles de potencia de la señal y del ruido respectivamente, estos valores se obtuvieron experimentalmente con el analizador de espectro y son: $\sigma_{S k}(d B)=1.816$ y $\sigma_{N}(d B)=0.8785 . Q(\cdot)$ es la función gaussiana $Q$ y $Q^{-1}(\cdot)$ es la inversa de $Q(\cdot)$.

$P_{R X k}$ es la potencia recibida por el usuario, la cual ya ha sido encontrada para el método propuesto en la ecuación (9). Mientras $P_{N}$, representa el piso de ruido del terminal de CR creado a partir de la suma de todas las fuentes de ruido en el receptor (incluyendo el ruido térmico), y se puede expresar como:

$P_{N}(d B m)=-174 \frac{d B m}{H z}+10 \log B(H z)+N F(d B)$

donde $-174 \mathrm{dBm} / \mathrm{Hz}$ es la densidad espectral de potencia de ruido térmico en $290^{\circ} \mathrm{K}$, B es el ancho de banda del canal detectado, NF es la figura de ruido total del receptor. La figura de ruido del amplificador de bajo ruido es $4 \mathrm{~dB}$ con una ganancia de $11 \mathrm{~dB}$, las pérdidas del cable son $0.72 \mathrm{~dB}$. La figura de ruido del analizador es $16 \mathrm{~dB}$ para la configuración realizada. Por tanto, la NF puede ser encontrada por el factor de ruido $\left(\mathrm{F}_{\mathrm{T}}\right)$ (Egan, 2003):

$F_{T}=F_{c a}+\frac{F_{L N A}-1}{G_{c a}}+\frac{F_{A n}-1}{G_{c a} G_{L N A}}=3.266$

$F_{c a}$ es el factor de ruido del cable, FLNA es el factor de ruido del amplificador de bajo ruido, $F_{\text {An }}$ es el factor de ruido del analizador de espectro, $\mathrm{G}_{\mathrm{ca}}$ es la ganancia del cable y GLNA es la ganancia del amplificador de bajo ruido. Por tanto, el NF es 5.14dB. El ciclo de trabajo, resultado de la ecuación (10), para el método propuesto en el sector de la celda de la BS del entorno exterior de la Fig. 2; se presenta en la figura 9.

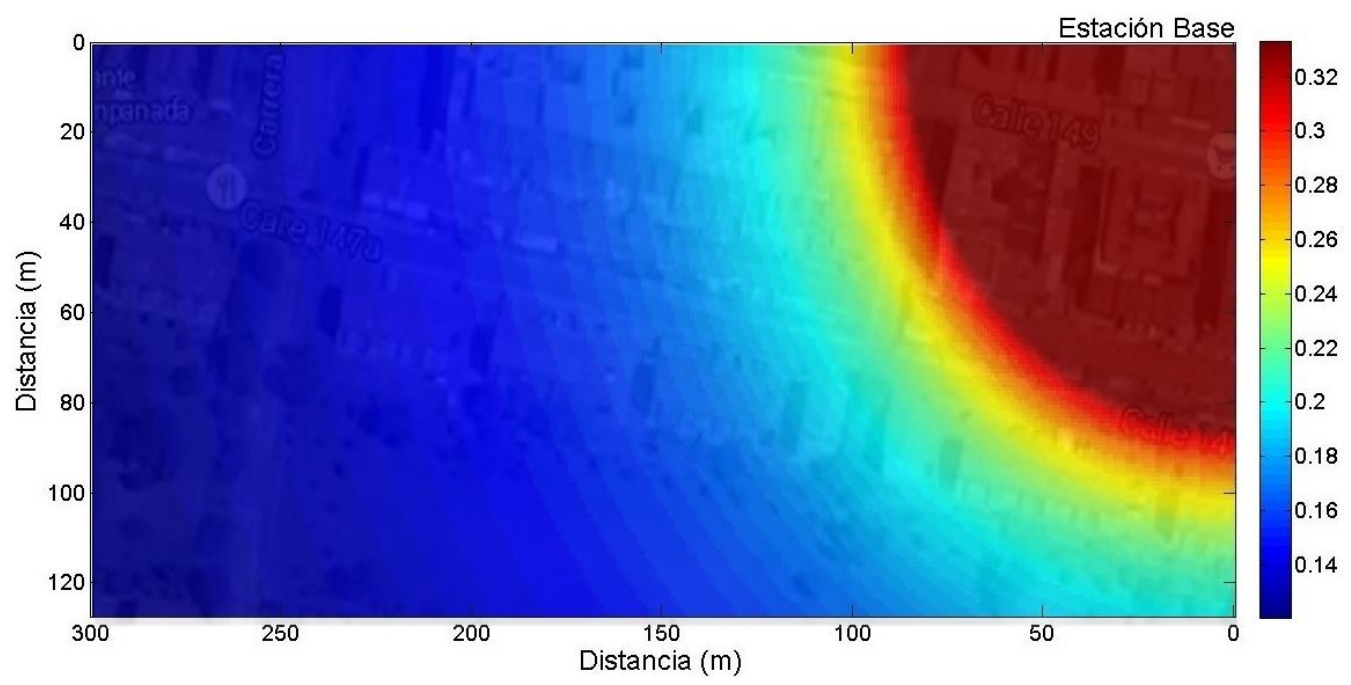

Fig. 9: Ciclo de trabajo para el método propuesto en el canal GSM 
En la Fig. 9 se observa que debido al planteamiento usado en la ecuación (10), en el escenario existen diferentes niveles de ocupación y no solo ocupado o libre, por ejemplo se puede encontrar que la probabilidad de ocupación del canal es baja o alta, pero no igual a cero o a uno. El modelamiento realizado es capaz de proporcionar una caracterización realista del pronóstico de la ocupación de espectro radioeléctrico en función del escenario de propagación considerado, que constituye un aspecto importante en el diseño y dimensionamiento de sistemas de CR para implementaciones reales. En la Fig. 9 se advierte también que los niveles de ocupación máxima oscilan alrededor de 0.32 . Estos valores corresponden a localizaciones cerca a la BS y son graficados con la gama de colores rojos. En general, los valores de ocupación van disminuyendo, y por tanto las oportunidades de espectro radioeléctrico para usuarios de CR se incrementan, a medida que la señal se aleja de la BS; y se representan mediante la gama de colores azules. Esto es coherente a nivel práctico y le da robustez al método propuesto.

\section{CONCLUSIONES}

En este estudio se desarrolló el diseño de un método para pronosticar las oportunidades de espectro radioeléctrico. Primero, a partir del ajuste del modelo de propagación SUI con las medidas realizadas en el entorno urbano. Posteriormente, dada la aproximación del ajuste con el promedio de los datos medidos, se integró con el modelo neuronal wavelet.

Se determinó las oportunidades de espectro radioeléctrico del método propuesto, a través del pronóstico de la potencia recibida en un tiempo determinado y del ciclo de trabajo a lo largo de un entorno urbano. Estos resultados demuestran su consistencia con el comportamiento práctico de los sistemas de comunicaciones móviles. La metodología propuesta presenta un enfoque novedoso y práctico para pronosticar la ocupación del espectro radioeléctrico que sería percibida por los usuarios de CR en escenarios reales.

La importancia en el pronóstico de la potencia recibida a través de los modelos de propagación radica en que los usuarios de CR pueden acceder a beneficios como: el ahorro de energía en el proceso de detección del espectro, aprovechar las oportunidades de espectro radioeléctrico incrementando la tasa de transmisión exitosa y las oportunidades de transmisión, reducir el tiempo para encontrar un canal disponible, y ajustar los niveles de potencia de transmisión para proteger de colisiones e interferencias a los usuarios primarios. Otra característica es que la mayor parte de los esquemas de pronóstico se ha basado en la determinación de huecos espectrales; mientras que este estudio se fundamenta en el conocimiento a priori de la potencia que se recibe por parte de un canal de los usuarios primarios, lo que permite evitar la selección de canales ruidosos y conllevar a un mejor reparto del espectro radioeléctrico entre los usuarios de CR. Esto permite lograr parámetros de calidad de servicio superiores con menos recursos de radio.

La CR es una de las tecnologías promisorias para la evolución del Internet de las cosas (IoT) en las comunicaciones inalámbricas. Por tanto, determinar las oportunidades de espectro radioeléctrico a través de modelos, como el desarrollado en este estudio, incrementa la cantidad de espectro radioeléctrico disponible y en consecuencia se aumenta la capacidad de interconexión de los dispositivos loT inalámbricos; aunque los dispositivos loT deben tener capacidades cognitivas para la detección y uso eficaz del espectro disponible.

\section{AGRADECIMIENTOS}

Los autores agradecen el apoyo del Centro de Investigaciones y Desarrollo Científico de la Universidad Distrital Francisco José de Caldas.

\section{REFERENCIAS}

Akyildiz, I., W.Y. Lee, M.C. Vuran y S. Mohanty, Next Generation / Dynamic Spectrum Access / Cognitive Radio Wireless Networks: A survey, doi: 10.1016/j.comnet.2006.05.001, Computer Networks Journal, 50, 2127-2159 (2006)

Al-Hourani, A., V. Trajkovi, S. Chandrasekharan y S. Kandeepan, Spectrum Occupancy Measurements for Different Urban Environments, European Conference on Networks and Communications, Paris (2015)

Bai, S., X. Zhou y F. Xu, "Soft Decision" Spectrum Prediction Based on Back-Propagation Neural Networks, International Conference on Computing, Management and Telecommunications, Da Nang (2014)

Bai, S., X. Zhou y F. Xu, Spectrum Prediction Based on Improved-Back-Propagation Neural Networks, International Conference on Natural Computation, Zhangjiajie (2015)

Chen, D., S. Yin, Q. Zhang, M. Liu y S. Li, Mining Spectrum Usage Data: A Large-Scale Spectrum Measurement Study, International Conference on Mobile Computing and Networking, Beijing (2009)

Chiang, R.I.C., G.B. Rowe y K.W. Sowerby, A Quantitative Analysis of Spectral Occupancy Measurements for Cognitive Radio, Vehicular Technology Conference, Dublin (2007) 
Debnath, L. y F. Shah, Wavelet Transforms and Their Applications, New York, Birkhäuser (2014) Egan, W.F., Practical RF System Design. New Jersey, Wiley (2003)

Erceg, V., L.J. Greenstein y otros cinco autores, An Empirically Based Path Loss Model For Wireless Channels in Suburban Environments, doi: 10.1109/49.778178, IEEE Journal on Selected Areas in Communications, 17(7), 12051211 (1999)

Galeano, K., L. Pedraza y D. Lopez, Spectrum Decision Model With Propagation Losses, ARPN Journal of Engineering and Applied Sciences, 11(21), 12786-12791 (2016)

Har, D., A.M. Watson y A.G. Chadney, Comment on Diffraction Loss of Rooftop-To-Street in COST 231-WalfischIkegami Mode, doi: 10.1109/25.790519, IEEE Transactions on Vehicular Technology, 48(5), 1451-1452 (1999)

Hata, M., Empirical Formula for Propagation Loss in Land Mobile Radio Services, doi: 10.1109/T-VT.1980.23859, IEEE Transactions on Vehicular Technology, 29(3), 317-325 (1980)

Haykin, S., Cognitive Radio: Brain-Empowered Wireless Communications, doi: 10.1109/JSAC.2004.839380, IEEE Journal on Selected Areas in Communications, 23(2), 201-220 (2005)

Hernández, C., L. Pedraza, I. Paéz y E. Rodriguez-Colina, Análisis de la Movilidad Espectral en Redes de Radio Cognitiva, doi: 10.4067/S0718-07642015000600018, Inf. Tecnol., 26(6), 169-186 (2015)

Iliya, S., E. Goodyer, M. Gongora, J. Shell y J. Gow, Optimized Artificial Neural Network Using Differential Evolution for Prediction of RF Power in VHF/UHF TV and GSM 900 Bands for Cognitive Radio Networks, Workshop on Computational Intelligence, Bradford (2014)

Kunwei, L., Z. Hangsheng, Z. Jianzhao, L. Cao y L. Menglin, A Spectrum Prediction Approach Based on Neural Networks Optimized By Genetic Algorithm In Cognitive Radio Networks, International Conference on Wireless Communications, Networking and Mobile Computing, Beijing (2014)

Lopez, M. y F. Casadevall, Methodological Aspects of Spectrum Occupancy Evaluation in the Context of Cognitive Radio, doi: 10.1002/ett.1453, European Transactions on Telecommunications, 21(8), 680-693 (2010)

Lopez, M. y F. Casadevall, Statistical Prediction of Spectrum Occupancy Perception in Dynamic Spectrum Access Networks, IEEE International Conference on Communications, Kyoto (2011)

López, D., E. Trujillo y O. Gualdron, Elementos Fundamentales que Componen la Radio Cognitiva y Asignación de Bandas Espectrales, doi: 10.4067/S0718-07642015000100004, Inf. Tecnol., 26(1), 23-40 (2015)

Lopez, M. y F. Casadevall, Space-Dimension Models of Spectrum Usage for Cognitive Radio Networks, doi: 10.1109/TVT.2016.2535903, IEEE Transactions on Vehicular Technology, 66(1), 306-320 (2017)

Madsen, K., H.B. Nielsen y O. Tingleff, Methods for Non-linear Least Squares Problems, Kongens Lyngby, Informatics and Mathematical Modelling, Technical University of Denmark (2004)

Mehdawi, M., N.G. Riley, M. Ammar, A. Fanan y M. Zolfaghari, Spectrum Occupancy Measurements and Lessons Learned in the Context of Cognitive Radio, Telecommunications Forum Telfor, Belgrade (2015)

Molisch, A.F., Wireless Communications, New York, Wiley (2011)

Okumura, Y., E. Ohmori, T. Kawano y K. Fukuda, Field Strength and its Variability in UHF and VHF Land-Mobile Radio Service, ISSN: 0142-3835, Review of the Electrical Communication Laboratory, 16(9), 825-873 (1968)

Ostlin, E., H.J. Zepernick y H. Suzuki, Macrocell Path-Loss Prediction Using Artificial Neural Networks, doi: 10.1109/TVT.2010.2050502, IEEE Transactions on Vehicular Technology, 59(6), 2735-2747 (2010)

Patil, K., K. Skouby, A. Chandra y R. Prasad, Spectrum Occupancy Statistics in the Context of Cognitive Radio, International Symposium on Wireless Personal Multimedia Communications, Brest (2011)

Pedraza, L., Modelo de Propagación para un Entorno Urbano que Identifica las Oportunidades Espectrales para Redes Móviles de Radio Cognitiva, Tesis Doctoral, Universidad Nacional de Colombia (2016)

Pedraza, L., F. Forero e I. Paez, Metropolitan Spectrum Survey in Bogota Colombia, IEEE International Conference on Advanced Information Networking and Applications Workshops, Barcelona (2013)

Pedraza, L., F. Forero e I. Paez, Evaluación de Ocupación del Espectro Radioeléctrico en Bogotá-Colombia, doi: 10.17230/ingciencia.10.19.6, Ingeniería y Ciencia, 10(19), 127-143 (2014)

Pedraza, L., C. Hernandez, K. Galeano, E. Rodríguez e I. Paez, Ocupación Espectral y Modelo de Radio Cognitiva para Bogotá, Bogotá, Universidad Distrital Francisco José de Caldas (2016a)

Pedraza, L., C. Hernandez y D. Lopez, A Model to Determine the Propagation Losses Based on the Integration of HataOkumura and Wavelet Neural Models, doi: 10.1155/2017/1034673, International Journal of Antennas and Propagation, 2017, 1-8 (2017)

Pedraza, L., C. Hernández e I. Páez, Evaluation of Nonlinear Forecasts for Radioelectric Spectrum, ISSN: 0975-4024, International Journal of Engineering and Technology, 8(3), 1611-1626 (2016b)

Pedraza, L., C. Hernández, I. Páez, J. Ortiz y E. Rodríguez-Colina, Linear Algorithms for Radioelectric Spectrum Forecast, doi: 10.3390/a9040082, Algorithms, 9(4), 1-18 (2016c) 
Pedraza, L., C. Hernandez y E. Rodriguez-Colina, Study of Models to Forecast the Radio-electric Spectrum Occupancy, doi: 10.17485/ijst/2016/v9i48/99370, Indian Journal of Science and Technology, 9(48), 1-19 (2016d)

Pedraza, L., C. Hernandez y E. Rodriguez-Colina, A Spectral Opportunities Forecasting Method in a Mobile Network Based on the Integration of COST 231 Walfisch-lkegami and Wavelet Neural Models, doi: 10.12988/ces.2017.68149, Contemporary Engineering Sciences, 10(3), 113-128 (2017a)

Pedraza, L., C. Hernández y O. Salcedo, Spectrum Forecast Using Propagation Losses of the Okumura-Hata Mode, doi: 10.15866/irecap.v6i5.10555, International Journal on Communications Antenna and Propagation, 6(5), 328-335 (2016e)

Phaiboon, S., P. Phokharatkul y S. Somkuarnpanit, 2 to $16 \mathrm{GHz}$ Microwave Line-of-Sight Path Loss Prediction on Urban Streets by Fuzzy Logic Models. Conference TENCON, Melbourne (2005)

Rappaport, T.S., Wireless Communications: Principles and Practice, New Jersey, Prentice-Hall (2002)

Rocke, S. y A.M. Wyglinski, Geo-Statistical Analysis of Wireless Spectrum Occupancy Using Extreme Value Theory, Conference on Communications, Computers and Signal Processing, Victoria (2011)

Sanders, F.H., Broadband Spectrum Survey at Los Angeles, California. U.S. Dept. of Commerce National Telecommunications and Information Administration, Boulder, Colo (1997)

Sarkar, T.K., J. Zhong, K. Kyungjung, A. Medouri y M. Salazar-Palma, A Survey of Various Propagation Models for Mobile Communication, doi: 10.1109/MAP.2003.123216, IEEE Antennas and Propagation Magazine, 45(3), 51-82 (2003)

Sotiroudis, S.P., S.K. Goudos, K.A. Gotsis, K. Siakavara y J.N. Sahalos, Application of a Composite Differential Evolution Algorithm in Optimal Neural Network Design for Propagation Path-Loss Prediction in Mobile Communication Systems, doi: 10.1109/LAWP.2013.2251994, IEEE Antennas and Wireless Propagation Letters, 12, 364-367 (2013)

Stocker, K.E., B.E. Gschwendtner y F.M. Landstorfer, Neural Network Approach to Prediction of Terrestrial Wave Propagation for Mobile Radio, doi: 10.1049/ip-h-2.1993.0048, IEE Proceedings H - Microwaves, Antennas and Propagation, 140(4), 315-320 (1993)

Taher, T.M., R.B. Bacchus, K.J. Zdunek y D.A. Roberson, Long-Term Spectral Occupancy Findings in Chicago, IEEE Symposium on New Frontiers in Dynamic Spectrum Access Networks, Aachen (2011)

Turin, G.L., F.D. Clapp, T.L. Johnston, S.B. Fine y D. Lavry, A Statistical Model of Urban Multipath Propagation, doi: 10.1109/T-VT.1972.23492, IEEE Transactions on Vehicular Technology, 21(1), 1-9 (1972)

Walfisch, J. y H.L. Bertoni, A Theoretical Model of UHF Propagation in Urban Environments, doi: 10.1109/8.14401, IEEE Transactions on Antennas and Propagation, 36(12), 1788-1796 (1988)

Wellens, M. y P. Mahonen, Lessons Learned from an Extensive Spectrum Occupancy Measurement Campaign and $A$ Stochastic Duty Cycle Mode, doi: 10.1109/TRIDENTCOM.2009.4976263, Mob. Netw. Appl., 15(3), $461-474$ (2010)

Zhang, Q. y A. Benveniste, Wavelet Networks, doi: 10.1109/72.165591, IEEE Transactions on Neural Networks, 3(6), 889-898 (1992) 
